\title{
Estimation of Generalized Logistic Distribution for Financial Data
}

\author{
Muhammad Idrees Ahmad \\ ${ }^{1}$ Sultan Qaboos University, Muscat, Oman
}

\begin{abstract}
Risk management of financial assets requires the knowledge of the form of the probability distribution to estimate the probability of extreme price changes. It is not only the shape of the distribution which is important but the methods of estimation also plays a fundamental role to compare the risk-reward tradeoff for different trading strategies with a reasonable adequacy. In the present study the generalized logistic distribution is considered to fit stock market returns of Muscat Securities Market. The extreme quantiles are estimated by the method of probability weighted moments and are compared with that of method of moments.
\end{abstract}

\section{Key words: Probability weighted moments, generalized logistic distribution, risk management, stock market.}

\section{INTRODUCTION}

The behavior of prices movements of stock process put significant influence on investors and financial managers on the choice and management of portfolio. Risk management of financial assets requires the knowledge of the form of the probability distribution to estimate the probability of extreme price changes [1]. It is not only the shape of the distribution which is important but the methods of estimation also plays a fundamental role to compare the risk-reward tradeoff for different trading strategies with a reasonable adequacy. In the present study the generalized logistic distribution is considered to fit stock market returns of Muscat Securities Market. The extreme quantiles are estimated by the method of probability weighted moments and are compared with that of method of moments.

The generalized logistic (GL) distribution came as investigated and accepts a lot of shapes of data to represent it in a better ways [2]. The (GL) distribution has three parameters and is also a special case of the kappa distribution. The cumulative distribution function (CDF) and the probability density function (PDF) of the GL distribution are defined respectively [3] as:

$$
F(x)=\frac{1}{1+e^{-y}}
$$

Pdf of the generalized logistic distribution is:
Where,

$$
f(x)=\frac{\alpha^{-1} e^{-(1-\beta) y}}{\left(1+e^{-y}\right)^{2}}
$$

$$
\begin{array}{ll}
y=-\beta^{-1} \log \left\{1-\frac{\beta\left(x-\mathrm{x}_{0}\right)}{\alpha}\right\}, & \text { if } \beta \neq 0 \\
y=\frac{x-\mathrm{x}_{0}}{\alpha}, & \text { if } \beta=0
\end{array}
$$

Where the inverse of the cdf is:

$$
\begin{array}{ll}
x(F)=x_{0}+\frac{\alpha\left[1-\left\{\frac{1-F}{F}\right\}^{\beta}\right]}{\beta}, & \text { if } \beta \neq 0 \\
x(F)=x_{0}-\alpha \log \left\{\frac{1-F}{F}\right\}, & \text { if } \beta=0
\end{array}
$$

Where $\mathrm{x}_{0}$ is the location parameter, $\alpha$ is the scale parameter, And $\beta$ is the shape parameter. The range of possible values for the $\mathrm{GL}$ distribution is given by: $\mathrm{x}_{0}+\frac{\alpha}{\beta} \leq x<$ $\infty$ for $\beta<0$; and

$-\infty<x \leq \mathrm{x}_{0}+\frac{\alpha}{\beta}$ for $\beta>0$.

If $\beta=0$ then the GL distribution is reduced to the 2parameter logistic distribution.

\section{METHODS ESTIMATION}

There are several methods that have been developed for estimating the parameters of a distribution, which are Method of Moments, Maximum

Corresponding Author: Muhammad Idrees Ahmad, Sultan Qaboos University, Muscat, Oman. idress@ squ.edu.om 
Muhammad Idrees Ahmad / Journal of Engineering and Science Research, 2 (2) 2018, Pages: 34-37

Likelihood Estimation, Simple Linear Estimation, Ordinary Least Square Method and Probability Weighted Moment. However, in this study, we will focus on methods of moments, and probability weighted moments.

\subsection{Method of Moments (MOM)}

We can estimate the parameters of generalized logistic distribution by using the Method of moments first to get the sample estimate of $\mu, \sigma^{2}, C_{s}$ and $C_{k}$ respectively where, $C_{s}$ and $\mathrm{C}_{\mathrm{k}}$ denote skewness and kurtosis and then equating them to their theoretical values. Let $\hat{\beta}, \hat{\alpha}$ and $\widehat{x_{0}}$ be the moments estimates of the parameters then the moment estimates will be as below [4].

$$
\begin{gathered}
\hat{\mu}=\hat{\mathrm{x}}_{0}+\frac{\hat{\alpha}}{\hat{\beta}}\left(1-g_{1}\right) \\
\hat{\sigma}^{2}=\frac{\hat{\alpha}^{2}}{\hat{\beta}^{2}}\left(g_{2}-g_{1}^{2}\right) \\
\widehat{C}_{s}=\hat{\gamma}_{1}=\frac{\hat{\mu}_{3}}{\hat{\mu}_{2}^{\frac{3}{2}}}=\frac{\hat{\beta}}{|\hat{\beta}|} \frac{-g_{3}+3 g_{1} g_{2}-2 g_{1}^{3}}{\left(g_{2}-g_{1}^{2}\right)^{2}} \\
\hat{C}_{k}=\hat{\gamma}_{2}=\frac{\hat{\mu}_{4}}{\hat{\mu}_{2}{ }^{2}}=\frac{\hat{\beta}}{|\hat{\beta}|} \frac{\left(g_{4}-3 g_{1}^{4}-4 g_{1} g_{3}+6 g_{1}^{2} g_{2}\right)}{\left(g_{2}-g_{1}^{2}\right)^{2}}
\end{gathered}
$$

Then equations will solved simultaneously and get these estimators:

$$
\begin{aligned}
& \hat{\beta}=\frac{2 \tan ^{-1}\left(-.59484 \hat{\gamma}_{1}\right)}{3 \pi} \\
& \widehat{\mathrm{x}_{0}}=\hat{\mu}-\{1-\Gamma(1+\hat{\beta}) \Gamma(1-\widehat{\beta})\} \\
& \hat{\alpha}=\frac{\hat{\sigma} \hat{\beta}}{\left\{\Gamma(1+2 \hat{\beta}) \Gamma(1-2 \hat{\beta})-\Gamma^{2}(1+\hat{\beta}) \Gamma^{2}(1-\hat{\beta})\right\}^{\frac{1}{2}}}
\end{aligned}
$$

\subsection{Probability Weighted Moments (PWM): These} estimate are obtained using the following formulae [5].

$$
\begin{gathered}
\widehat{\beta}=\frac{-\hat{\lambda}_{3}}{\hat{\lambda}_{2}} \\
\hat{\alpha}=\frac{\hat{\lambda}_{2}}{\Gamma(1-\hat{\beta}) \Gamma(1+\hat{\beta})} \\
\hat{\mathrm{x}}_{0}=\hat{\lambda}_{1}-\frac{\widehat{\alpha}\{1-\Gamma(1-\hat{\beta}) \Gamma(1+\hat{\beta})\}}{\hat{\beta}}
\end{gathered}
$$

Where $\lambda_{r}=r^{-1} \sum_{k=0}^{r-1}(-1)^{k}\left(\begin{array}{c}r-1 \\ k\end{array}\right) E X_{r-k: r} r=1,2, \ldots$. $\lambda_{r}$ is a linear function of the expected order statistics, defined $\alpha_{r}$ and $\mathrm{B}_{r}$, we have

$$
\begin{gathered}
\lambda_{r+1}=(-1)^{r} \sum_{k=0}^{r} p_{r, k}^{*} \alpha_{k}=\sum_{k=0}^{r} p_{r, k}^{*} \mathrm{~B}_{k}, r=1,2, \ldots . \\
\text { where } \\
p_{r, k}^{*}=(-1)^{r-k}\left(\begin{array}{l}
r \\
k
\end{array}\right)\left(\begin{array}{c}
r+1 \\
k
\end{array}\right)
\end{gathered}
$$

We estimate $\mathrm{B}_{r}$ by $b_{r}=n^{-1} \sum_{i=1}^{n} \frac{(i-1)(i-2) \ldots \ldots(i-r) X_{j}}{(n-1)(n-2) \ldots \ldots .(n-r)}$ in order to get $\lambda_{r},[6]$.

\section{DATA}

In the present study we took the monthly closing values of indices for the entire composite index over the period from October 2009 to October 2015 providing a total of 74 observations. We brought these data from the website of the Muscat Securities Market. This data includes indices' price of the main index. The primary interest for the investors is to have information about return they will get from their investment. For this reason we worked out the percentage rather than the indices. The percentage returns are computed for the monthly closing returns over a 6 years [1].

Return $=\frac{(\text { curent } \text { close }- \text { previous close })}{\text { previous close }} * 100 \%$

\subsection{The Results}

The estimates of the parameters along with upper and lower quantile estimates by each method of estimation are presented in table 1. The MOM gives the estimates of quantiles much higher at the upper tail of the distribution.

Table 1 estimate parameters and quantiles of PWM \& MOM estimators

\begin{tabular}{|c|r|r|}
\hline Method & PWM & MOM \\
\hline$\hat{\beta}$ & 0.23849 & 0.13715 \\
\hline$\hat{\alpha}$ & 1.71279 & 1.80219 \\
\hline$x_{0}$ & 0.56105 & 0.25791 \\
\hline$\widehat{x_{.99}}$ & 5.34240 & 6.40133 \\
\hline$\widehat{x_{.95}}$ & 4.18439 & 4.62367 \\
\hline$\widehat{x_{.90}}$ & 3.49024 & 3.67676 \\
\hline$\widehat{x_{.1}}$ & -4.38579 & -4.36329 \\
\hline$\widehat{x_{.05}}$ & -6.75170 & -6.28004 \\
\hline$\widehat{x_{.01}}$ & -13.74409 & -11.27961 \\
\hline
\end{tabular}


Muhammad Idrees Ahmad / Journal of Engineering and Science Research, 2 (2) 2018, Pages: 34-37

The corresponding probabilities of exceedances are given in table .2. These probabilities are the probabilities of Value at risk.

Table 2: return period and value at risk of estimate quantiles of PWM \& MOM estimators.

\begin{tabular}{|c|c|c|c|c|}
\hline $\begin{array}{c}\text { Waiting } \\
\text { time } \\
\text { (T=111- } \\
\text { F) }\end{array}$ & $\begin{array}{c}\text { Probability } \\
\text { of } \\
\text { exceedance }\end{array}$ & quantile & PWM & MOM \\
\hline 100 & .01 & $\widehat{x_{.99}}$ & 5.3424 & 6.4013 \\
\hline 20 & .05 & $\widehat{x_{.95}}$ & 4.1843 & 4.6236 \\
\hline 10 & .1 & $\widehat{x .90}$ & 3.4902 & 3.6767 \\
\hline 1.1 & .9 & $\widehat{x_{.1}}$ & -4.3857 & -4.3632 \\
\hline 1.05 & .95 & $\widehat{x_{.05}}$ & -6.7517 & -6.2800 \\
\hline 1 & .99 & $\widehat{x_{01}}$ & -13.7440 & -11.2796 \\
\hline
\end{tabular}

The Value at Risk (VAR) calculates the maximum loss expected (or worst case scenario) on an investment, over a given time period and given a specified degree of confidence. VaR is widely applied in finance for quantitative risk management for many types of risks.. This value can be given by the following probability inequality for example

$$
p(x \leq \widehat{x .01})=.01
$$

and so on with other quantiles. This can also be expressed in terms of Return period.

We will consider the return period for the quantile of $1 \%, 5 \%, 10 \%, 90 \%, 95 \%$, and $99 \%$ for both probability weighted moments, and method of moments. The period will be calculated by equation $1 / 1-F$ where $\mathrm{F}$ is the quantile.

By using PWM for 0.99 the return period will be 1/1$.99=100$ this means that after 100 months the probability of gaining the investment is 5.34240 . And for 0.95 the return period will be $1 / 1-.95=20$ this means that after 20 months the probability of gaining the investment is 4.18439 . For 0.90 the return period will be $1 / 1-.90=10$ this means that after 10 months the probability of gaining the investment is 3.490243 . On the other hand 0.1 the return period will be $1 / 1-.1=1.1$ this means that after 1.1 months the probability of losing the investment is 4.3857 . Also for 0.05 the return period will be $1 / 1-.05=1.05$ this means that after 1.05 months the probability of losing the investment is 6.7517. Finally 0.01 the return period will be $1 / 1-.01=1$ this means that after 1 months the probability of losing the investment is 13.7440 .

By using MOM after 100 months the probability of gaining the investment is 6.4013 in 0.99 . And after 20 months the probability of gaining the investment is 4.6236 in 0.95 . In 0.90 the probability of gaining the investment is 3.6236 after 10 months. On the other hand after 1.1 months the probability of losing the investment is 4.3632 in 0.1 . Also for 0.05 the probability of losing the investment is 6.2800 after 1.05 months. Finally in 0.01 the probability of losing the investment is 11.2796 after one month.

\section{CONCLUSION}

The empirical distribution of returns from Muscat Stock Market is investigated. It is observed that the distribution of the returns of closing index in MSM is not normal. It has a negative skewness of -1.29 and positive kurtoses of 1.86 above normal. More over the Anderson Daring test rejects the hypothesis of the normality. Therefor we considered the generalized logistic distribution as an alternative in this study. This distribution can also take into account to a negative skewness.

The parameters of this distribution are estimated by probability weighted moments and by method of moments. The extreme quantiles at the upper as well as at the lower tails of the distribution were worked out by each method. Generally the estimate of the quantiles by MOM is higher than that of by the PWM. The average waiting time of various durations was also estimated by each method. It is noticed that we have to wait on average 100 months to get return greater than or equal to $5.342 \%$ using PWM while this would be $6.4013 \%$ if estimated by MOM. However as the waiting time decreases the percentage return becomes similar by each method for both methods.

\section{References}

[1] Ahmad, M. I. (2009). Fitting Extreme Value Type I Distribution to Financial Returns. Journal of Material Science and Engineering, USA. Volume 3, Number 10, p83-86, October 2009.

[2] Quanxi Shao (2002) . MAXIMUM LIKELIHOOD ESTIMATION FOR GENERALISED LOGISTIC DISTRIBUTIONS, Communications in Statistics - Theory and Methods, 31:10, 1687-1700, DOI: 10.1081/STA120014908 
Muhammad Idrees Ahmad / Journal of Engineering and Science Research, 2 (2) 2018, Pages: 34-37

[3] M. O. Ojo and A. K. Olapade(2003). ON THE GENERALIZED LOGISTIC AND LOG-LOGISTIC DISTRIBUTIONS, Department of Mathematics, Obafemi Awolowo University, Ile-Ife, Nigeria

[4] Muna R.Alkasasbeh,Mohammad Z.Raqab (2009)Estimation of the generalized logistic distribution parameters Comparative study.. Statistical Methodology 6 (2009) 262_279
[5] Hosking, J.R.M. (1986). The Theory of Probability Weighted Moments, Research Report RC12210, IBM Research Division, Yorktown Heights,

[6] Hosking, J.R.M. (1990). "L-moments: Analysis and estimation of distribution using linear combinations of order statistics." Journal of the Royal Statistical Society, Series B, $52,105-124$ 